\title{
Metallic phase of disordered graphene superlattices with long-range correlations
}

\author{
Hosein Cheraghchi, ${ }^{1,}$ * Amir Hossein Irani, ${ }^{1}$ Sayyed Mahdi Fazeli, ${ }^{2}$ and Reza Asgari ${ }^{3}$, \\ ${ }^{1}$ School of Physics, Damghan University of Basic Sciences, 6715- 364, Damghan, Iran \\ ${ }^{2}$ Physics Department, the University of Qom, Qom, Iran \\ ${ }^{3}$ School of Physics, Institute for Research in Fundamental Sciences, IPM 19395-5531 Tehran, Iran
}

(Dated: November 9, 2018)

\begin{abstract}
Using the transfer matrix method, we study the conductance of the chiral particles through a monolayer graphene superlattice with long-range correlated disorder distributed on the potential of the barriers. Even though the transmission of the particles through graphene superlattice with white noise potentials is suppressed, the transmission is revived in a wide range of angles when the potential heights are long-range correlated with a power spectrum $S(k) \sim 1 / k^{\beta}$. As a result, the conductance increases with increasing the correlation exponent values gives rise a metallic phase. We obtain a phase transition diagram in which a critical correlation exponent depends strongly on disorder strength and slightly on the energy of the incident particles. The phase transition, on the other hand, appears in all ranges of the energy from propagating to evanescent mode regimes.
\end{abstract}

PACS numbers: 73.23.-b,73.63.-b

\section{INTRODUCTION}

The exploration of graphene, a monolayer of carbon atoms tightly packed into a honeycomb lattice, has been recently attracted special attentions for investigation of the fundamental physics and also probable device applications such as nanoelectronic based on planar graphene structures 1.2 . In graphene, due to its unique band structure with the valence and conduction bands touching at two inequivalent Dirac points electrons around the Fermi level obey the massless relativistic Dirac equation, which results in a linear energy dispersion relation ${ }^{3}$. Massless relativistic quasiparticles arising from the cone spectrum lead to a number of unusual electronic properties such anomalous integer ${ }^{4}$ and fractiona $l^{5}$ quantum Hall effects, focusing of electron by a rectangular potential barrier (Veselago lensing) ${ }^{6}$, special Andreev reflection ${ }^{7}$, observation of the plasmaron composite ${ }^{8}$ and minimal conductivity $\stackrel{9}{\text {. }}$.

Interestingly, relativistic quantum quasiparticles incident normally to a high electrostatic potential barrier in graphene can pass through it with perfect transmission regardless of the height and width of the barrier ${ }^{10}$. This phenomenon which is referred as the Klein tunneling is in contrast with the quantum massive carrier tunneling where the transmission probability decays exponentially with increasing of the barrier height and width. Recently, evidences for the Klein tunneling of the Dirac fermions across p-n junction have been experimentally observed when a gate-induced potential step is steep enough ${ }^{11,12}$.

In graphene sheets the type of particle (electrons or holes) and the density of the carriers can be controlled by tuning a gate bias voltage $\mathrm{e}^{13}-15$. Moreover, graphene superlattices may be fabricated by adsorbing adatoms on the graphene surface by positioning and aligning impurities with scanning tunneling microscopy 16 , or by applying a local top gate voltage to graphene $\frac{17}{18}$. The transition of hitting massless particles in a clean $\frac{18}{}$ or disordered ${ }^{19}$ graphene-based superlattice structure has been studied.

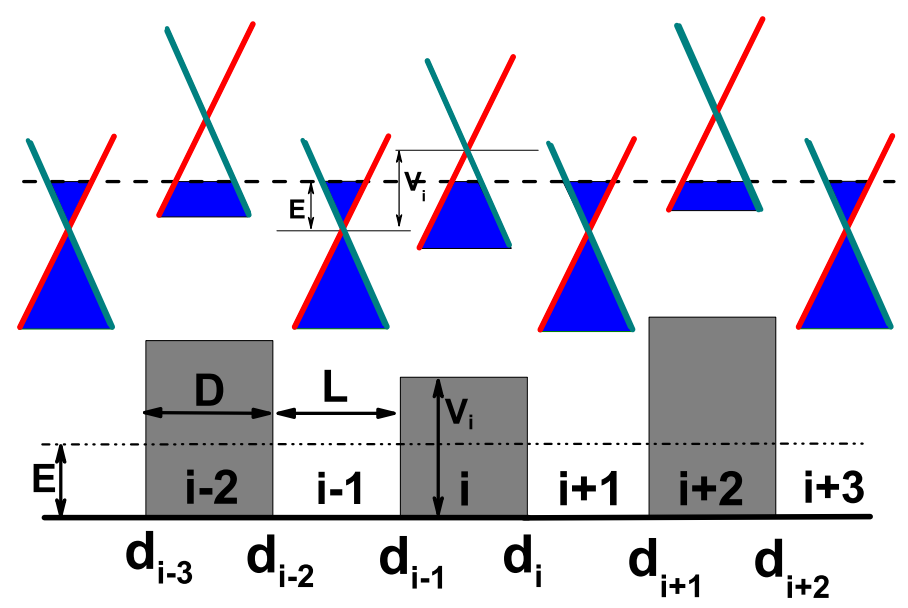

FIG. 1: Graphene superlattice with long-range correlated disorder on the potential barriers.

It is shown that the conductivity of the system depends on the superlattice structural parameters.

The first study on electronic properties of monolayer and bilayer graphene superlattices was performed by Bai and Zhang 18 . They showed that the angularly averaged conductivity can be controlled by changing the structure parameters. It has been shown that massless Dirac fermions are generated in one-dimensional external periodic potential close to the original Dirac point ${ }^{20,21}$. The Dirac points depend on geometrical parameters for instance the potential of the barriers/wells, the period of the potential and transverse wave number ${ }^{22}$. An evidence for such Dirac points is the conductance resonances are appeared in the special potential values 21 . Moreover, the conductance of graphene superlattice with uncorrelated disorder on the width of the barriers was calculated in [19]. It was shown that the transmission of the quasiparticles with large angles incidence to the potential bar- 
riers is suppressed by disorder strength and the sample size too. Therefore, the results of the finite-size scaling computations predicted a zero conductance for all the graphene superlattices, except for some resonant barrier thickness in which the conductance tends to a nonzero constant in the thermodynamic limit $\underline{19}$.

A number of numerical calculations of electron transport confirmed the absence of the localization in the presence of the long-range random potential in disordered graphene $e^{23}$. The main quantity mostly studied numerically is the conductance, $G$ of a finite-size graphene sample with a width, $W$ much larger than the length, $L$. The setup allows us to define the "conductivity" $\sigma=G L / W$ even for ballistic samples with $L$ much shorter than the mean free path, $l$.

It is well known that the transmission of the quantum massive carriers unexpectedly increases when special correlation is applied on disorder ${ }^{24}$. This is in contrast with the Anderson localization in which all states are exponentially localized in one dimensional uncorrelated disorder. Experimental evidence for discrete the number of the extended states has been observed in random-dimer semiconductor superlattices as a short-range correlated disorder ${ }^{25}$. Long-range correlated sequences of the potential barriers in semiconductor superlattices, however, could result in a continuum of extended states giving rise the mobility edges $26-28$.

In this paper, we study the conductance of massless Dirac fermions through graphene superlattices with a long-range correlated disorder on the potentials of the barriers. Transmission of the large angles incident electrons to graphene superlattice which are suppressed by uncorrelated disorder, are revived by applying correlation between random potentials of the barriers. As a result, the conductance increases with the correlation exponent. Consequently, an insulator-to-metal transition emerges at a critical correlation exponent which depends strongly on the disorder strength. One should notice that such phase transition emerges for all ranges of the energy. In addition, the dependence of the conductance to the superlattice parameters is investigated for different correlation strengths.

The paper is organized as follows: we present the transfer matrix and Fourier Filtering method used to calculate transmission through long-range correlated graphene superlattices in Section II. Our results and discussions will be presented in Section IV presenting the metal-toinsulator phase transition and investigating the emergence of the phase transition along different energy ranges. The last Section concludes our results.

\section{MODEL AND THE TRANSFER MATRIX}

In the low-energy limit, charge carriers near the Dirac point in the continuum model can be described by the following non-interacting Hamiltonian

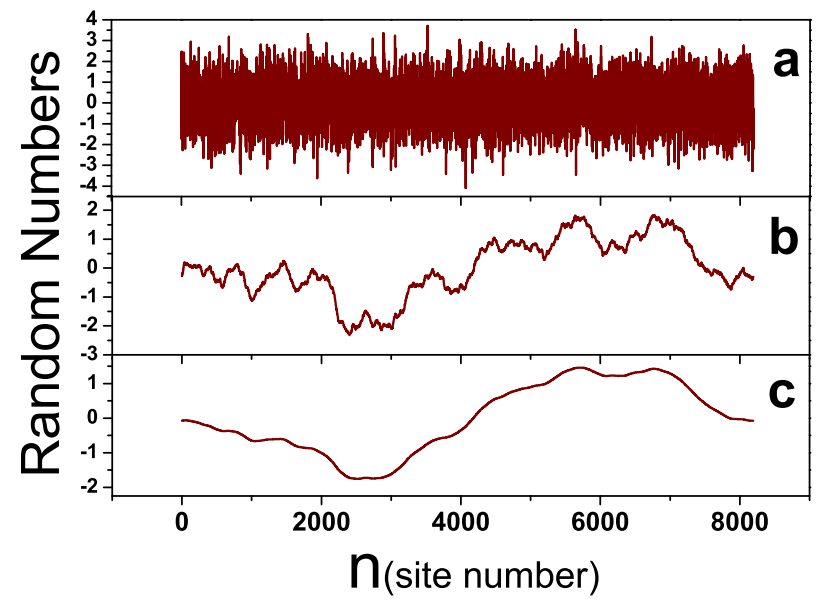

FIG. 2: Random distribution of the correlated sequences generated by Fourier Filtering Method. a) uncorrelated case corresponding to $\alpha=0.500, \mathrm{~b}) \alpha=1.766$, c) $\alpha=1.993$.

$$
H=-i \hbar v_{\mathrm{F}} \vec{\sigma} \cdot \vec{\nabla}+V(x)
$$

where $v_{\mathrm{F}}=10^{6} \mathrm{~m} / \mathrm{s}$ is the Fermi velocity and $\vec{\sigma}=$ $\left(\sigma_{x}, \sigma_{y}\right)$, are Pauli matrices. We consider a lattice of the electrostatic potentials as barriers which are induced by top gate voltages. Therefore, the potential of the barriers are sorted as the following:

$$
V(x)=\left\{\begin{array}{cc}
V_{i} & d_{2 i-1}<x<d_{2 i}, \quad i=1,2, \ldots \\
0 & \text { elsewhere }
\end{array}\right.
$$

A schematic representation of graphene superlattice is shown in Fig. 1. We consider the number of regions (wells and barriers) to be equal $N$ and then there is $(N-1) / 2$ barriers. The width of the barriers and wells are considered to be fixed. The height of the barriers fluctuates around its mean is defined by $V_{i}=\langle V\rangle\left(1+\sigma \varepsilon_{i}\right)$ where $\sigma$ is the variance of the potentials of the barriers and $\left\{\varepsilon_{i}\right\}$ is a long-range correlated random sequence of data with the Gaussian distribution. The random sequences will become normalized, accordingly the mean value $\varepsilon_{i}$ is set to be zero and its variance has been fixed.

Before passing to calculate the conductance, we might generate a random sequence with long-range correlation, $\left\{\varepsilon_{i}\right\}$ will be considered to describe the trace of a fractional Brownian motion with a power spectrum $S(k) \sim 1 / k^{\beta}$ where $1<\beta<3$ and $\beta=2 H+1=2 \alpha-1(1<$ $\alpha<2)$. Here $H$ is the Hurst exponent. In the case of power-law decaying auto-correlations, the correlation function decays with an exponent $\gamma$ such that $C\left(x_{i}-\right.$ $\left.x_{j}\right) \propto|i-j|^{-\gamma}$ where $\gamma=1-\beta$. Random sequences with weaker positive correlation is generated with $0.5<\alpha<1$ which is referred to fractional Gaussian noise ${ }^{30}$. In this case, $\beta=2 H-1=2 \alpha-1$ and $\alpha=0.5$ corresponds to uncorrelated disorder or white-noise. 


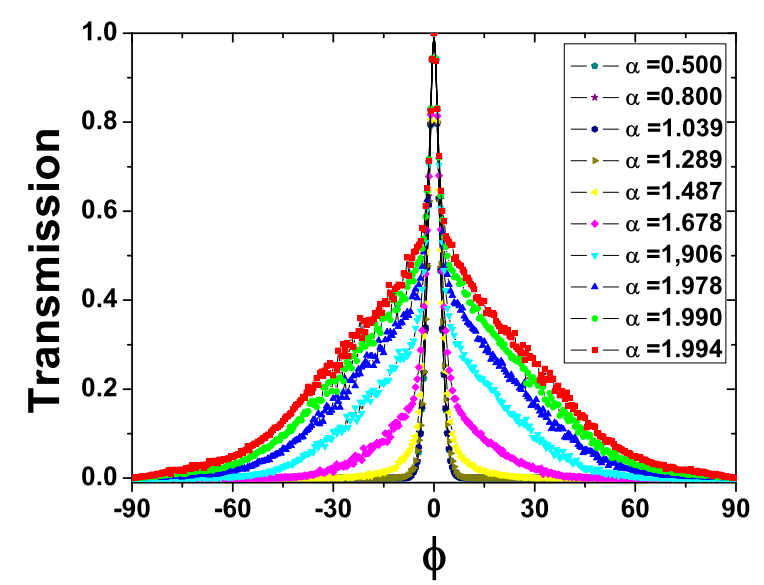

FIG. 3: Transmission in terms of the incident angle hitting to graphene superlattice with various correlation exponents $\alpha$. The number of the barriers is $N=1000$. Averaged potential of the barriers and energy of the incident electrons are $\langle V\rangle=200 \mathrm{meV}$ and $E=50 \mathrm{meV}$, respectively. Here, since $\xi=-3$, and transmission is calculated in the presence of purely propagating modes.

It is commonly usual to apply Fourier filtering $\operatorname{method}^{29}$ to generate a sequence with the long-range correlation. The method is based on a transformation of the Fourier components $\left(\theta_{k}\right)$ of a random number sequence $\left\{\theta_{i}\right\}$. Uncorrelated random numbers $\left\{\theta_{i}\right\}$ have a Gaussian distribution. Finally, the inverse Fourier transformation of the sequence $\left\{\varepsilon_{k}\right\}\left(=k^{-\frac{\beta}{2}} \theta_{k}\right)$ results to the interested sequence $\left\{\varepsilon_{i}\right\}$. Three landscapes of random data generated by the mentioned method are shown in Fig. 2 for different correlation exponents. Clearly, correlation between random potentials leads to a reduction in the fluctuations of the random distribution. We should notice that we have checked the invariance of our results in comparison with those results obtained from random sequences produced by the mid-point method ${ }^{30}$.

Having such configuration of the potentials gives rise a superlattice consists of two types of graphene as electrondoped or hole-doped. The doping type of graphene in wells is n-type for quasiparticles with incident energy $E=h v_{\mathrm{F}} / \lambda>0$ where $\lambda$ is electron wavelength in wells. The doping type in barriers depends on the potential height $V_{i}$. In other words, the $i^{\text {th }}$ barrier is hole-doped graphene if $E<V_{i}$, while it is electron-doped if $E>V_{i}$. Accordingly, the type of doping in barriers is also altered randomly. We assume that the angle of electrons incidence to superlattice is $\phi=\varphi_{1}$ along the $x$ axes. However, the transfer matrix takes the angle of each region into account separately. The general solution of Eq. (11) results in the following spinor for the $i^{\text {th }}$ region;

$$
\begin{array}{r}
\psi(x, y)=a_{i}\left\{\begin{array}{c}
1 \\
s_{i} e^{i \varphi_{i}}
\end{array}\right\} e^{i\left(k_{i x} x+k_{y} y\right)} \\
+b_{i}\left\{\begin{array}{c}
1 \\
s_{i} e^{i\left(\pi-\varphi_{i}\right)}
\end{array}\right\} e^{i\left(-k_{i x} x+k_{y} y\right)}
\end{array}
$$

where $a_{i}$ and $b_{i}$ are the transmission and reflection amplitudes, respectively. Other parameters in the spinor are

$$
\begin{array}{r}
s_{i}=\operatorname{sgn}\left(E-V_{i}\right), \quad k_{y}=k_{\mathrm{F}}^{i} \sin \left(\varphi_{i}\right)=k_{\mathrm{F}}^{i} \sin \phi \\
k_{i x}=\sqrt{\left((E-V(x)) / \hbar v_{\mathrm{F}}\right)^{2}-k_{y}^{2}}, \quad \varphi_{i}=\arctan \left(k_{y} / k_{i x}\right) \cdot(4)
\end{array}
$$

If the energy of the incident electrons being close to $V_{i}$ value in the $i^{\text {th }}$ barrier, $k_{i x}$ will become imaginary value in some angles, resulting in an evanescent mode. In disordered graphene superlattice, evanescent modes emerge when $E \simeq\langle V\rangle$. The transfer matrix is extracted by the continuity of the wave functions at the junction interfaces. It can make a relation between wave functions of two sides of a step potential from the $i^{\text {th }}$ region to the $(i+1)^{\text {th }}$ like

$$
\left(\begin{array}{c}
a_{i+1} \\
b_{i+1}
\end{array}\right)=M_{i+1, i}\left(\begin{array}{c}
a_{i} \\
b_{i}
\end{array}\right)
$$

where the transfer matrix $M_{i+1, i}$ is;

$$
M_{i+1, i}=\left(\begin{array}{ll}
m_{11} & m_{12} \\
m_{12}^{*} & m_{11}^{*}
\end{array}\right)
$$

and the matrix elements of $M$ are as follows;

$$
\begin{aligned}
& m_{11}=e^{i\left(k_{i x}-k_{(i+1) x}\right) x_{i}}\left(\frac{s_{i+1} e^{-i \varphi_{i+1}}+s_{i} e^{i \varphi_{i}}}{2 s_{i+1} \cos \varphi_{i+1}}\right) \\
& m_{12}=e^{-i\left(k_{i x}+k_{(i+1) x}\right) x_{i}}\left(\frac{s_{i+1} e^{-i \varphi_{i+1}}-s_{i} e^{-i \varphi_{i}}}{2 s_{i+1} \cos \varphi_{i+1}}\right)
\end{aligned}
$$

The current in the $x$-direction and in the $i^{\text {th }}$ region can be derived as the following;

$$
J_{x}^{i}=v_{\mathrm{F}} \psi^{\dagger} \sigma_{x} \psi=2 v_{\mathrm{F}} s_{i} \cos \varphi_{i}\left(\left|a_{i}\right|^{2}-\left|b_{i}\right|^{2}\right)
$$

Current conservation between regions $i^{\text {th }}$ and $j^{\text {th }}$ implies on

$$
\left|a_{j}\right|^{2}-\left|b_{j}\right|^{2}=\operatorname{Det}\left[M_{j, i}\right]\left(\left|a_{i}\right|^{2}-\left|b_{i}\right|^{2}\right)
$$

where

$$
\begin{array}{r}
M_{j, i}=M_{j, j-1} M_{j-1, j-2} \ldots M_{i+1, i} \\
\operatorname{Det}\left[M_{j, i}\right]=s_{i} \cos \varphi_{i} / s_{j} \cos \varphi_{j}
\end{array}
$$




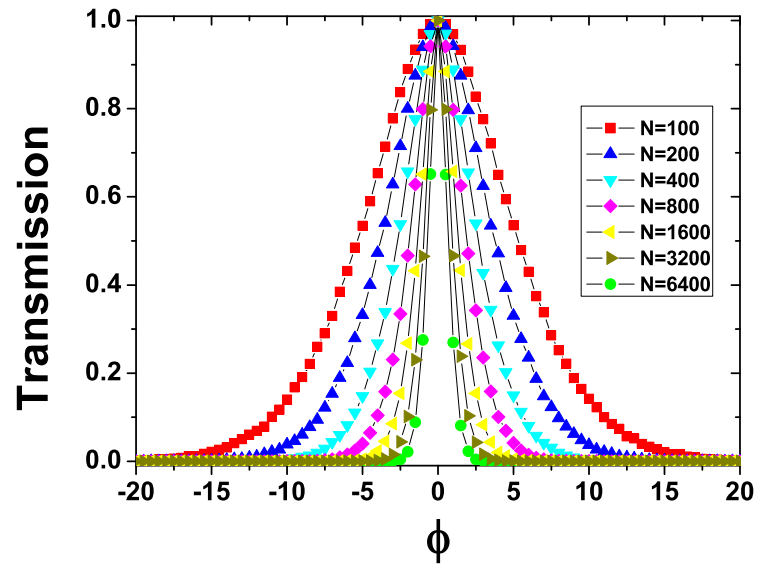

FIG. 4: Transmission as a function of the incident angle through graphene superlattice with long-range correlated random potentials for various barrier numbers $N$. Here $\alpha=1.102$ and $\sigma=0.1$.

The total transfer matrix which makes a relation between incident and transmitted wave functions is a series product of the transfer matrices arising from each interface. For $N$ regions incorporating of the barriers and wells, regarding to Eq.(11), the matrix is defined as $P=M_{N, 1}$.

If the first and last regions of superlattice are electrondoped graphene, transmission probability for $(N-1) / 2$ barriers can be calculated by means of the product matrices for $b_{N}=0$ as the following

$$
T(E, \phi)=\frac{J_{o u t}^{N}}{J_{\text {in }}^{1}}=\frac{1}{\operatorname{Det}[P]}\left|\frac{a_{N}}{a_{1}}\right|^{2},
$$

where $J_{\text {out }}^{N}$ and $J_{\text {in }}^{1}$ are out- and inflowing currents, respectively. Because the configuration of the potential barriers is considered such a way that $s_{1}=s_{N}$ and $\varphi_{1}=\varphi_{N}=\phi$, the conservation of the current between the first and last regions implies that $\operatorname{Det}[P]=1$. Therefore, the transmission formula can be simplified as $T(E, \phi)=1 /\left|P_{22}\right|^{2}$ where $a_{N} / a_{1}=\operatorname{Det}[P] / P_{22}$. Finally, using Landauer-Büttiker formula 31 and an angularly averaging, the conductance is obtained by the following integration.

$$
G=G_{0} \int_{-\pi / 2}^{\pi / 2} T(E, \phi) \cos (\phi) d \phi
$$

where $G_{0}=e^{2} m v_{\mathrm{F}} W / \hbar^{2}$. Here $W$ is the finite width of graphene ribbon along the $y$-direction.

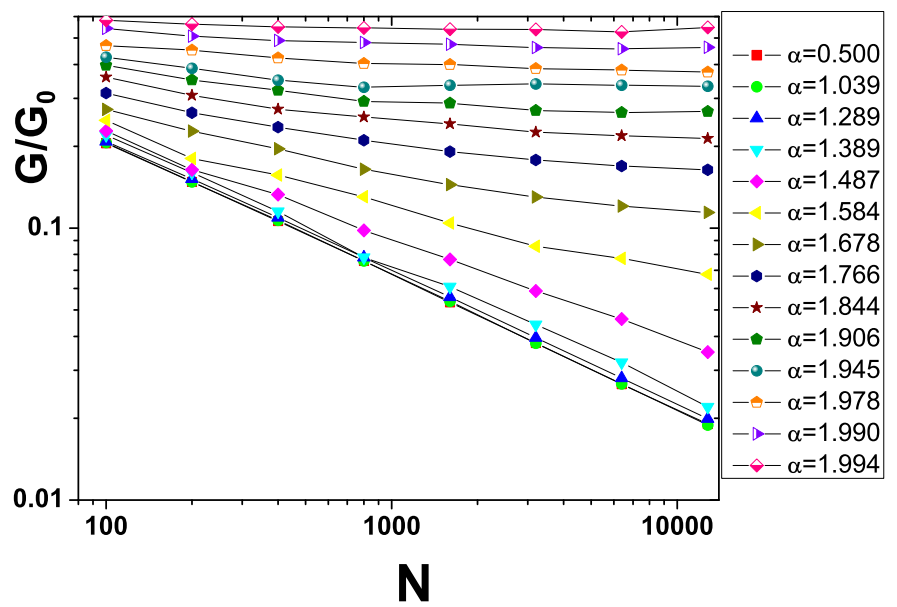

FIG. 5: Conductance through graphene superlattice with long-range correlated random potentials as a function of barrier number $N$ for $\sigma=0.1$. This is a finite size scaling for different correlation exponents $\alpha$. Here, $\langle V\rangle=200 \mathrm{meV}$ and $E=50 \mathrm{meV}$.

\section{RESULTS AND DISCUSSION}

\section{A. Phase transition}

Let us first calculate the transmission probability and study the electronic properties of disordered graphene superlattices. The transmission of electrons hitting to a disordered graphene superlattice as a function of the incident angle is shown in Fig. 3 for several values of correlation strengths characterizing with the correlation exponent $\alpha$. In all calculations, barrier and well widths are considered to be $D=50 \mathrm{~nm}$ and $L=30 \mathrm{~nm}$, respectively. Moreover, we assumed that the energy of the charge carriers and the averaged potential of barriers being $E=50 \mathrm{meV}$ and $\langle V\rangle=200 \mathrm{meV}$, respectively. The wavelength of the incident electrons is thus $\lambda \cong 83 \mathrm{~nm}$. Therefore, with such parameters we surely conclude that the transmission of the charge carriers shown in Fig. 3 (with $\xi=(E-\langle V\rangle) / E=-3)$ is a purely propagating mode. As shown in this figure, the transmission of the electrons hitting to superlattice with large angles increases by increasing correlation between the random potentials of the barriers. In other words, applying correlation between random potentials of the barriers causes to extend the angular window of the conducting mode around the normal incidence. This effect is in contrast with those results obtained in uncorrelated potentials of the barriers in which the transmission of the massless carriers is suppressed for the large incident angles except at $\phi=0$. Perfect transmission at normal incidence can be described by the Klein tunneling. Similarly, in the correlated case, by increasing disorder strength and also the number of the barriers, the transmission of the quasiparticles is suppressed at all ranges of the incident angle except at $\phi=0$. Fig. 4 shows the suppression of the 


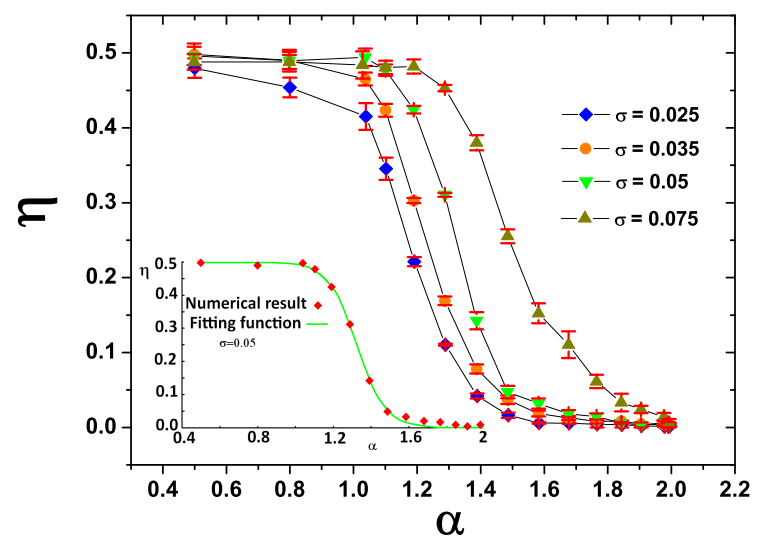

FIG. 6: Exponent of the conductance in a power law form $G / G_{0} \propto N^{-\eta}$ as a function of correlation exponent $\alpha$. In the inset the fitting of a Fermi-Dirac like function (Eq14) to numerical data is shown.

transmission at large incident angles when the number of the potential barriers increases.

In linear regime, the conductance is proportional to angularly averaged transmission projected along the current direction. To understand how the correlation between random potentials affects on the transport properties of graphene superlattice, we calculated the size dependence of the conductance for the various values of the correlation exponents and results are plotted in Fig. 5. It is clear from the figure that there is a critical correlation exponent value of $\alpha$ such that for $\alpha<\alpha_{c r}$, the conductance decreases with increasing system size, while it goes to a constant value for $\alpha>\alpha_{c r}$. In other words, a consequence of applying long-range correlation is the emergence of a phase transition from the insulating to metallic states. It is worthwhile noting that this phase transition is not a finite size effect. The conductance decreases with the number of the barriers as a power law behavior. The following function is fitted to a log-log plot of the conductance.

$$
\frac{G}{G_{0}} \propto N^{-\eta(\alpha, \sigma)}
$$

where $\eta$ as functions of $\alpha$ and $\sigma$ decreases by increasing the correlation exponent. Furthermore, disorder strength strongly suppresses the conductance such that the critical correlation exponent which implies on the emergence of a phase transition, increases with the disorder strength.

Conductance through disordered potentials of barriers having correlation exponent $\alpha=0.5$ decays with the barriers number as $N^{-0.5}$. The same decaying of the conductance was reported in Ref. [19] for graphene superlattice with white noise potentials distributed on the barriers. However, applying a long-range correlation between random potentials facilitates the conductance

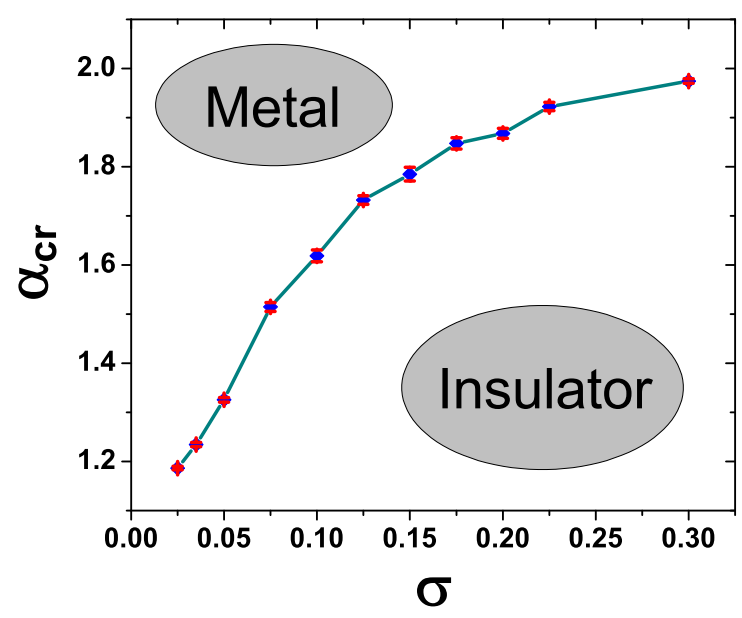

FIG. 7: Metal-to-insulator phase diagram. The critical correlation exponent, $\alpha_{c r}$ increases with disorder strength, $\sigma$.

through graphene superlattice.

A power-law fitting for the conductance is shown in Fig. 5. It is determined a dependency of $\eta$ on the correlation exponent $\alpha$ and disorder strength $\sigma$ which is represented in Fig. 6. For the sake of having the critical correlation exponent function, we have used a fit function of the exponent function $\eta(\alpha, \sigma)$ in a transition function such as a Fermi-Dirac function:

$$
\eta(\alpha, \sigma)=\frac{\gamma}{e^{\beta\left(\alpha-\alpha_{c r}(\sigma)\right)}+1} \rightarrow\left\{\begin{array}{cc}
0 & \alpha>>\alpha_{c r} \\
\gamma & \alpha<<\alpha_{c r}
\end{array}\right.
$$

where $\beta$ and $\alpha_{c r}$ are fitted parameters and $\gamma=0.5$ for $\xi=$ -3 . The range that $\eta(\alpha)$ decreases from the value of $\gamma$ to zero is related with the inverse of $\beta$. The emergence of a transition from insulating to metallic phase corresponds to the variation of $\eta$ from $\gamma$ to 0 . Now, we provide a phase transition diagram in which the critical correlation exponent depends on disorder strength. Fig. 7 shows that the critical correlation exponent increases when disorder strength increases up to $\sigma=0.3$. Roughly speaking, the system remains in a metallic phase when the correlation is very long range at low disorder strength whereas it turns out to be an insulator at large disorder strength values and at mid-range correlation.

\section{B. Energy range of the phase transition}

To provide a fascinating experimental manifestation of the phase transition, it is significant to demonstrate that the phase transition can exist in a continuum range of energies not just at some discrete energies.

Let us now concentrate on the conductance behavior in the different range of the Fermi energy. For a sin- 


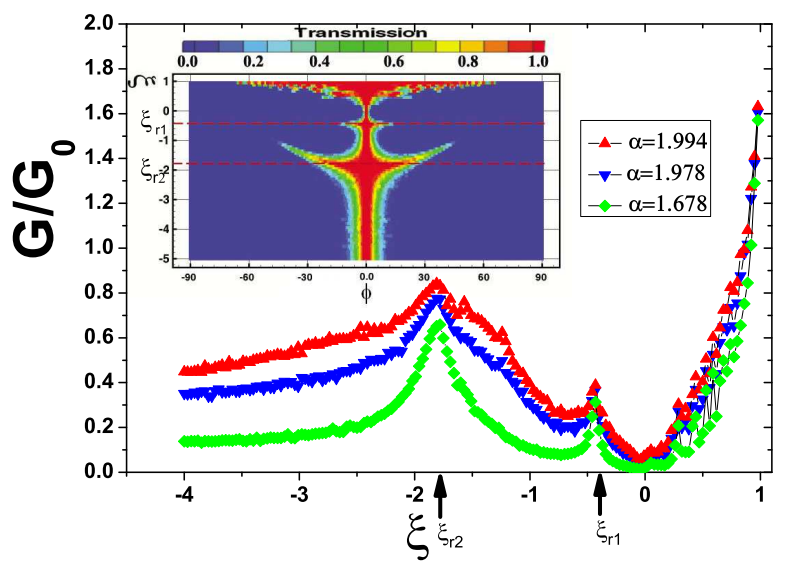

FIG. 8: Functional of the conductance in terms of $\xi$ which represents Fermi energy. There are two peaks in conductance correspond to resonant states. In the inset $3 \mathrm{D}$ contour plot of the transmission in the plane of $\xi$ and the incident angle. Resonant states corresponds to more angularly open a domain for transport. Here $N=800$ and $\sigma=0.1$.

gle barrier on graphene, in the range of $-1<\xi<1$, it is proved that both the evanescent and propagating modes coexist 32 . Out of this range, all states are fully in the propagating modes. By considering this fact, we investigate the conductance as a function of $\xi$ through a disordered graphene superlattice for several correlation exponent values and the results are shown in Fig. 8, It can be seen that the same behavior as a single barrier case is appeared in the different ranges of $\xi$. Contribution of the evanescent modes in the conductance is dominant in $\xi=0$ and therefore the conductance is suppressed in this point. For $|\xi| \rightarrow 1$, the contribution of the propagating modes becomes dominant and thus the conductance increases. For $\xi<0$, the conductance oscillates at resonant states which originates from the perfect tunneling of the charge carriers near to a normal incidence. A 3D contour plot of the transmission in terms of $\xi$ and the incident angle (see the inset Fig. 8) shows that at resonant states, a conducting domain of the angles is opened around the normal incidence. The resonant condition for a single barrier in graphene is given by $k_{\mathrm{F}} D \sqrt{\xi^{2}-u^{2}}=n \pi$ where $u=\sin \varphi$. Distance of the resonant states can be extracted from expanding the resonant condition for normal incidence $u \ll \xi$. In this case, two sequential resonant states have a distance like $\Delta \xi_{r} \cong \frac{\pi}{k_{\mathrm{F}} D}\left(1-\frac{u^{2}}{2 \xi^{2}}\right)$. As a result, the period of the conductance reduces with $D, L$ while enhances with $|\xi|$. In the limit of $\xi \rightarrow 1$ which it means $E \gg\langle V\rangle$, the conducting channels are opened for the whole range of the angles, $T(\phi)=1$, and thus by using Eq. (12), we then get $\lim _{\xi \rightarrow 1} G / G_{0}=2$.

By applying a long-range correlation between the potentials of the barriers, Fig. 8 indicates that the conductance shows an enhancement in some ranges of the energy compared to the resonant states and the range

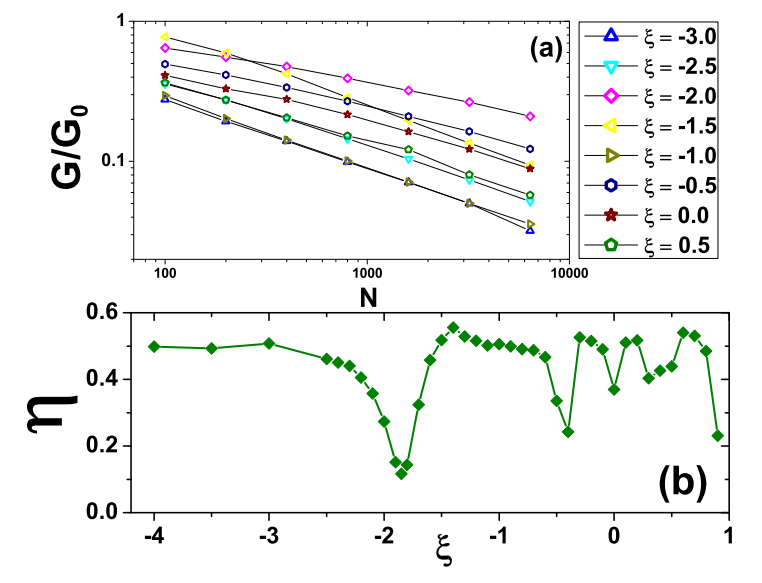

FIG. 9: a) Conductance in terms of the barrier numbers for graphene superlattice with white-noise disorder on potentials of the barriers and for different values of the Fermi level $\xi$. b) Exponent of the conductance $(\eta)$ in a power-law form $G / G_{0} \propto$ $N^{-\eta}$ as a function of $\xi$.

including the evanescent modes $|\xi|<1$. For clarifying that, firstly we investigate the conductance suppression with the number of the barriers over all ranges of the Fermi energy for a sequence of white noise disorder distributed on the potentials of the barriers. Results are represented in Fig. 9a which show a power-law form of the conductance in terms of the barrier numbers for different values of $\xi$. It is significant to understand how the exponent of $\eta$ varies with the Fermi energy. The slope of the lines in log-log plot of Fig. 9a for different $\xi$ are indicated in Fig. 9b. It is clear that close to resonant states $\xi_{r}=-1.85,-0.45$, the exponent of $\eta$ reduces from the value of 0.5 to its resonant values $\eta_{r}=0.12,0.25$, respectively. As a consequence, close to resonant states, the suppression of the conductance which is induced by random potentials of the barriers is much weaker than other states. The resonant value of $\eta_{r}$ decreases when $\xi$ goes away from the region including the evanescent modes $|\xi|<1$.

Our numerical calculations demonstrate that the insulator-to-metal phase transition occurs in all ranges of the energy. It is clear from Fig. 10 that the phase transition appears not only at the propagating and resonant states, but also in the fully evanescent mode $\xi=0$. In Fig. 10, $\eta(\alpha=0.5)$ decreases to 0.3 at $\xi=-2$ which is close to the second resonant state shown in Fig. 8. Another exception around the resonant states is the width of function $\eta(\alpha)$ which increases at the resonant states. Therefore, at the resonant states, the transition from insulating to metallic phase is smooth along the correlation exponent. The inset Fig. 10 shows small fluctuations of the critical correlation exponent as a function of $\xi$. Accordingly, the phase transition is a general behavior of all ranges of the energy. 


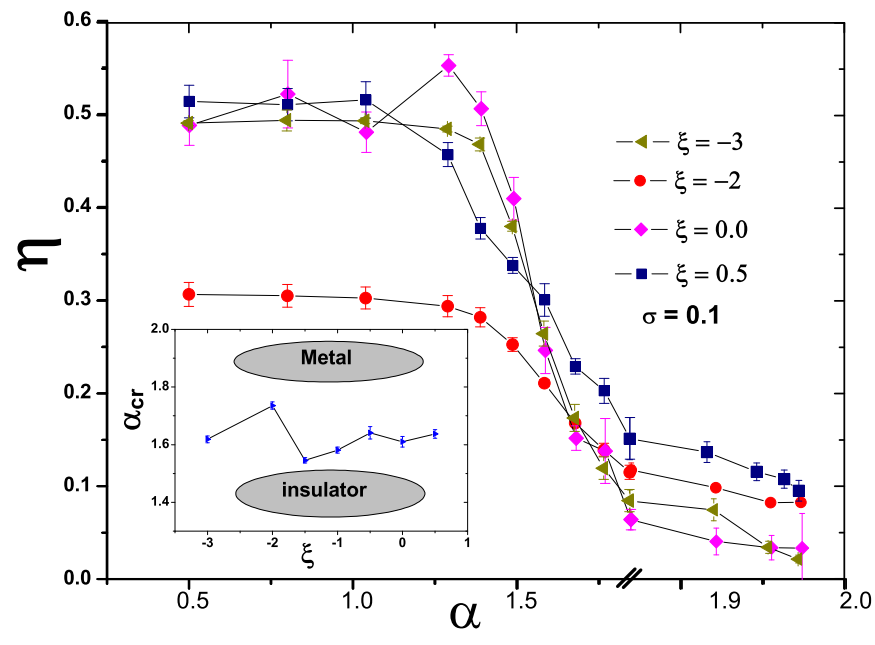

FIG. 10: Exponent of the conductance in a power law form $G / G_{0} \propto N^{-\eta}$ as a function of the correlation exponent $\alpha$ for different values of $\xi$. In the inset the metal-to-insulator phase transition in all ranges of the energy.

\section{Resonance in the conductance}

Now, we study the effect of the long-range correlated disorder on the resonance phenomena seen in the conductance. Resonance in the conductance of graphene superlattice with white noise disorder distributed on width of the barriers has been studied before $\underline{19}$. Fig. 11 shows the conductance oscillations as a function of the barrier width $(D)$ and also distance between barriers $(L)$ for several values of the correlation exponent. Apparently, the application of the long range correlation between potentials of the barriers increases the conductance for all ranges of $D$ and $L$. Moreover, as shown in Fig. 11], the conductance tends to a constant value after some oscillations in thin barriers independent of the barrier width. In fact, the transmission of the quasiparticles hitting to graphene superlattice at large incident angles is suppressed for the wide barriers, and thus only transmission arising from the Klein tunneling around the normal incident contributes into the conductance integration given by Eq. (12). The same behavior occurs in the case of $N=1$. Configuration average of the transmission through one barrier ${ }^{33}$ depends on the width by functions of $<\sin ^{2}\left(k_{x} D\right)>_{\text {C.A. }}$. and $<\cos ^{2}\left(k_{x} D\right)>_{C . A}$. where $k_{x}$ is a random parameter. It is trivial that the average of the transmission and consequently the conductance become independent of the width when $D$ value increases. On the other hand, by applying of the randomness on $k_{x}$, the resonant condition $k_{x} D=n \pi$ in wide barriers can not be satisfied, and there is thus no longer the resonant peak in the conductance.

Fig. 11b shows the conductance oscillations as a function of $L$. In this case, the decaying of the oscillations is much weaker than the conductance oscillations with the barrier width. In fact, since there is no disorder in the wells, in a fixed barrier width, the resonant condition affects much less than the barrier resonant condition.
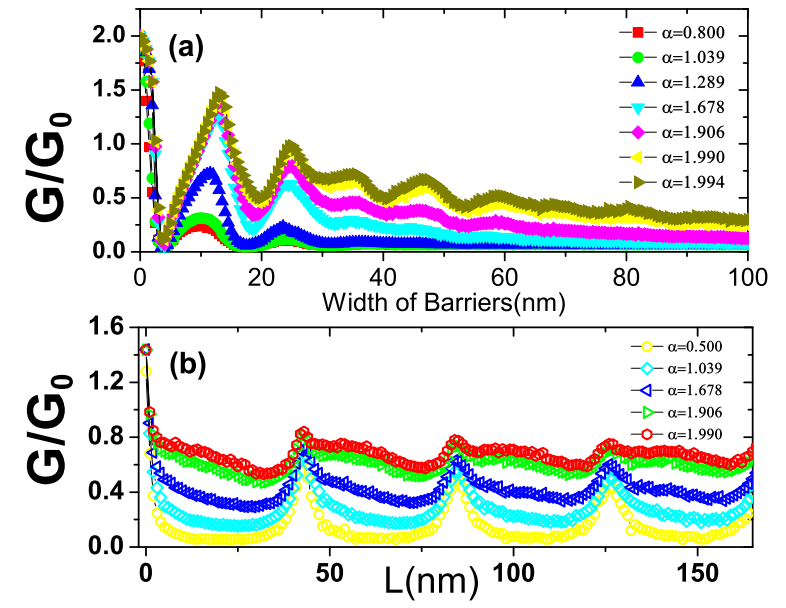

FIG. 11: Conductance oscillations in terms of a) the width $(D)$ of the barriers b) the distance $(L)$ between barriers for different correlation exponents. Here $N=800$ and $\sigma=0.1$.

\section{CONCLUSION}

By using the transfer matrix method, we investigate the conductance through a graphene superlattice with long-range correlated disorder distributed on potentials of the barriers. Applying a correlation between potentials opens angularly domain window of the conducting channels in competition with the factor of the disorder strength which suppress the transmission at large incident angles. As a result, the conductance increases with the correlation between the potentials of the barriers gives rise a metallic phase. We obtain a phase transition diagram in which the critical correlation exponent for such a phase transition depends strongly on disorder strength and slightly on the energy of the incident particles. At resonant states, the suppression of the conductance with the number of the barriers is much less than other states. Our finding for the dc conductance of the graphene superlattices should be important to the design of electronic nanodevices based on graphene superlattices.
* Electronic address: cheraghchi@dubs.ac.ir

$\dagger$ Electronic address: asgari@ipm.ir

${ }^{1}$ K. S. Novoselov, A. K. Geim, S. V. Morozov, D. Jiang, Y.
Zhang, S. V. Dubonos, I. V. Grigorieva, and A. A. Firsov, Science 306, 666 (2004)

2 A. Geim, Science 324, 1530(2009); 
3 R. Wallace, Phys. Rev. 71, 622 (1947). T. Ando, J. Phys. Soc. Jpn. 74, 777 (2005).

4 V. P. Gusynin and S. G. Sharapov, Phys. Rev. Lett. 95, 146801 (2005).

5 X. Du, I. Skachko, F. Duerr, A. Luican, and E.Y. Andrei, Nature 462, 192 (2009); K.I. Bolotin, F. Ghahari, M.D. Shulman, H.L. Stormer, and P. Kim, Nature 462 , 196 (2009) .

6 V. V. Cheianov, V. Falko, and B. L. Altshuler, Science 315, 1252 (2007).

7 C. W. J. Beenakker, Phys. Rev. Lett. 97, 067007 (2006) .

8 A. Bostwick, F. Speck, T. Seyller, K. Horn, M. Polini, R. Asgari, A. H. MacDonald and E. Rotenberg, Science 328, 999 (2010)

${ }^{9}$ K. S. Novoselov, A. K. Geim, S. V. Morozov, D. Jiang, M. I. Katsnelson, I. V. Grigorieva, S. V. Dubonos, and A. A. Firsov, Nature 438, 197 (2005); ibid: Nat. Phys. 2, 177 (2006).

10 O. Klein, Z. Phys. 53, 157165 (1929).

11 J. R. Williams, L. DiCarlo, and C. M. Marcus, Science 317, 638(2007) .

12 N. Stander, B. Huard, and D. Goldhaber-Gordon, Phys. Rev. Lett. 102, 026807 (2009) .

13 K.S. Novoselov, A.K. Geim, S.V. Morozov, D. Jiang, M.I. Katsnelson, I.V. Grigorieva, S.V. Dubonos, A.A. Firsov, Nature 438, 197 (2005) .

14 Y.B. Zhang, Y.W. Tan, H.L. Stormer, P. Kim, Nature 438, $201(2005)$

15 J. Nilsson, A.H. Castro Neto, F. Guinea, N.M.R. Peres, Phys. Rev. B 76, 165416 (2007).

16 H. Hiura, Appl. Surf. Sci. 222, 374 (2004) ; J.C. Meyer, C.O. Girit, M.F. Crommie, A. Zettl, Appl. Phys. Lett. 92 123110 (2008) .

17 B. Huard, J.A. Sulpizio, N. Stander, K. Todd, B. Yang, D. Goldhaber-Gordon, Phys. Rev. Lett. 98, 236803 (2007).

18 C. Bai and X. Zhang, Phys. Rev. B 76, 075430 (2007).

19 N. Abedpour, Ayoub Esmailpour, Reza Asgari, and M. R. Tabar, Phys. Rev. B, 79, 165412 (2009).

20 C.-H. Park, Y.-W Son, L. Yang, M.L. Cohen, and S.G.
Louie, Phys. Rev. Lett. 103, 046808 (2009) .

21 L. Brey and H.A. Fertig, Phys. Rev. Lett. 103, 046809 (2009) .

22 M. Barbier, P. Vasilopoulos, and F. M. Peeters, Phys. Rev.

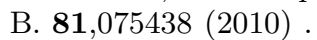

${ }^{23}$ K. Nomura and A.H. MacDonald, Phys. Rev. Lett. 98, 076602 (2007); A. Rycerz, J. Tworzydlo, and C.W.J. Beenakker, Europhys.Lett. 79, 57003 (2007); J.H. Bardarson, J. Tworzydlo, P. W. Brouwer, and C.W.J. Beenakker, Phys. Rev. Lett. 99, 106801 (2007). K. Nomura, M. Koshino, and S. Ryu, Phys. Rev. Lett. 99, 146806 (2007); P San-Jose, E. Prada, and D.S. Golubev, Phys. Rev. B 76, 195445 (2007).

24 D. H. Dunlap, H. L. Wu, P.W. Phillips, Phys. Rev. Lett, 65, 88 (1990).

25 V. Bellani, E. Diez, R. Hey, L. Toni, L. Tarricone, G. B. Parravicini, F. Dominguez-Adame, and R. Gomez-Alcala, Phys. Rev. Lett.82, 2159 (1999) .

26 F. A. B. F. deMoura, M. L. Lyra, Phys. Rev. Lett. 81, 3735 (1998) .

27 H. Cheraghchi, S. M. Fazeli, and K. Esfarjani, Phys. Rev. B. 72, 174207 (2005) .

28 A. Esmailpour, M. Esmaeilzadeh, E. Faizabadi, P. Carpena, and M. R. Tabar, Phys. Rev. B 74, 024206 (2006) .

29 H.A. Makse, S.Havlin, M. Schwartz, and H.E. Stanley, Phys. Rev. E, 53, 5445 (1996) .

30 Heinz-Otto Peitgen, Dietmar Saupe, "The Science of Fractal Images", Springer-Verlag New York, Inc. New York, NY, USA (1988) .

31 S. Datta, Electronic Transport in Mesoscopic Systems ( Cambridge University Press, London, 1995) .

32 H. Haugen, D. Huertas-Hernando, and A. Brataas, Phys. Rev. B, 77, 115406 (2008).

33 A. H. Castro Neto, F. Guinea, N. M. R. Peres, K. S. Novoselov, and A. K. Geim, Rev. Mod. Phys. 81, 109 (2009) . 